\title{
Defining the position of deep inguinal ring in patients with inguinal hernia
}

\author{
De Silva KPVR ${ }^{1}$, Hettiarrachchi $\mathbf{M}^{2}$, Tilakeratne $\mathbf{S}^{\mathbf{1}}$ \\ ${ }^{1,3}$ Teaching Hospital Karapitiya, Galle, ${ }^{2}$ Nuclear Medicine Unit, Faculty of Medicine, Galle, Sri Lanka \\ Correspondence to: Dr K.P.V.R De Silva (kpvrdesilva@yahoo.com)
}

\begin{abstract}
Introduction: Identification of the exact position of the deep inguinal ring is important in classifying inguinal hernias. This information is often used in evaluating short cases in undergraduate as well as postgraduate examinations in Surgery.

Methods: Consenting patients undergoing routine inguinal herniotomy and mesh repair in a Surgical Unit of the Teaching Hospital Karapitiya were included in the study. The length between the anterior superior iliac spine (ASIS) and the pubic tubercle (PT) was measured using a sterilized metal ruler.

Results: Twenty five patients participated in this study. Fifteen had right inguinal hernia and others had left inguinal hernia. The mean distance from the PT to the ASIS in those with left inguinal hernia (LIH) was $6.61 \mathrm{~cm}$. $(\mathrm{SD}=0.34 \mathrm{~cm})$. The mean distance from the left PT to the actual position of the deep inguinal ring was $3.05(0.14)$ $\mathrm{cm}$. In patients who had right inguinal hernia the mean distance from the PT to the right ASIS was $6.65(0.32)$ $\mathrm{cm}$. The mean distance from the right PT to the actual position of the deep inguinal ring was $3.07(0.15) \mathrm{cm}$.
\end{abstract}

Conclusion: The exact position of the deep inguinal ring is medial to the mid-point of the inguinal ligament. Our data reveal that it is not lying in the mid-point of the inguinal ligament.

Key words: Deep inguinal ring, inguinal hernia

\section{Introduction}

Identification of the exact position of the deep inguinal ring (DIR) is important in performing various clinical procedures and in diagnosing certain hernias. The method of identifying the position of deep inguinal ring is to take the midpoint between anterior superior iliac spine (ASIS) and pubic tubercle (PT) (1) i.e., the midpoint of inguinal ligament. According to our observations, preoperative estimates of the position of the DIR are not accurate measures of its true position in patients with inguinal hernias. Clinical evaluation of inguinal hernias, either pre-operative or during examinations, based on this traditional teaching may lead to misclassification of inguinal hernias. Therefore, we planned a study to assess the actual position of deep inguinal ring among Sri Lankan subjects.

\section{Methods}

Consecutive patients undergoing routine inguinal herniotomy and mesh repair in our surgical unit, Teaching Hospital Karapitiya, were included in the study. The length between anterior superior iliac spine (ASIS) and pubic tubercle (PT) was measured using a sterilized metal ruler. Weight of each individual was measured using the standard ward scale. Informed written consent was obtained from the patients before including them in the study.

In all cases, a transverse incision over the lower abdominal skin crease was made and then it extended two third of the way from the midline to the ASIS. Next, the superficial fascia was incised to expose the external oblique aponeurosis. This was followed by a short split with the knife in the line of the fibers of the external oblique aponeurosis over the inguinal canal. This split was then enlarged in medial and lateral directions and then mobilized the cord. 
The cord was then dislocated laterally and downwards to expose a direct hernia. To exclude an indirect sac spermatic fascia was opened to identify the edge of the peritoneum at the deep inguinal ring. Once the position of the DIR was confirmed by identifying the extraperitoneal fat around the deep inguinal ring, the distance between the ASIS and PT was measured. All the measurements were made by one person to minimize inter-observer variation. If the direct hernia was detected, hernial sac was reduced and strengthening of the posterior wall by uniting the conjoint tendon into inguinal ligament was performed. Finally posterior wall was strengthened by fixing a polypropylene mesh.

\section{Data analysis}

Data were tabulated and the exact positioning of the DIR and its association to the mid-point of the inguinal ligament was determined. The differences between the left and right sided inguinal hernia patients in the assessment of mid-point assessments were analyzed using independent sample t-test. Pearson's correlation (r) was used to determine the correlations between these parameters. Two-tailed $\mathrm{p}<0.05$ was considered to be statistically significant. Statistical analysis was done using SPSS version 15.0 (SPSS Inc., Chicago, USA).

Table: Basic characteristics of the study sample ${ }^{1}$

\section{Results}

There were 25 male patients participated in this study. The age of these patients ranged from 30.0 to 78.0 years with an average of 55.80 (SD 12.5) years. Fifteen $(\mathrm{n}=15)$ patients had right inguinal hernia (RIH) while others had left inguinal hernia (LIH). The mean age of presentation and the weight of these subjects were not significantly different when compared with the side of hernia presentation (Table). The mean (SD) distance from the PT to the ASIS (i.e. the length of the canal) in those with LIH was $6.61(0.34) \mathrm{cm}$. The mean distance from the left PT to the actual position of the deep inguinal ring (exact position of deep ring) was $3.05(0.14) \mathrm{cm}$.

In patients who had RIH, the mean length of the canal was $6.65(0.32) \mathrm{cm}$. The exact position of deep ring, was $3.07(0.15) \mathrm{cm}$ (Table). It was also interesting to note that the actual position of the DIR was medial to the midpoint between the PT \& ASIS in all the subjects who participated in this study. Furthermore, there was a linear relationship with the 'midpoint' (between PT \& ASIS) and the 'deviation from the midpoint' among these subjects $(r=0.40$; $\mathrm{p}=0.05)$; Figure). There was a significant positive correlation between age of the patients with length of the canal $(r=0.40 ; p=0.05)$ and the exact position of deep ring $(r=0.44 ; p=0.03)$. Further, length of the canal had significant positive correlation with the exact position of deep ring $(r=0.92 ; p<0.001)$.

\begin{tabular}{lll}
\hline Parameter & \multicolumn{2}{c}{ Inguinal Hernia } \\
& Left $(\mathbf{n}=\mathbf{1 0})$ & Right $(\mathbf{n}=\mathbf{1 5})$ \\
\hline Age (year) & $52.50(12.55)$ & $58.00(12.44)$ \\
Weight $(\mathrm{kg})$ & $66.40(3.92)$ & $67.47(4.34)$ \\
Length of canal (cm) & $6.61(0.34)$ & $6.65(0.32)$ \\
Midpoint $(\mathrm{cm})$ & $3.31(0.17)$ & $3.32(0.16)$ \\
Exact position of deep ring $(\mathrm{cm})$ & $3.05(0.14)$ & $3.07(0.15)$ \\
Deviation from the midpoint $(\mathrm{mm})$ & $2.55(0.83)$ & $2.50(0.46)$ \\
\hline
\end{tabular}

${ }^{1}$ There were 25 patients (all males) in the study. Results presented as mean (SD). None of these measurements were significantly different between groups (left vs. right sided inguinal hernia) 


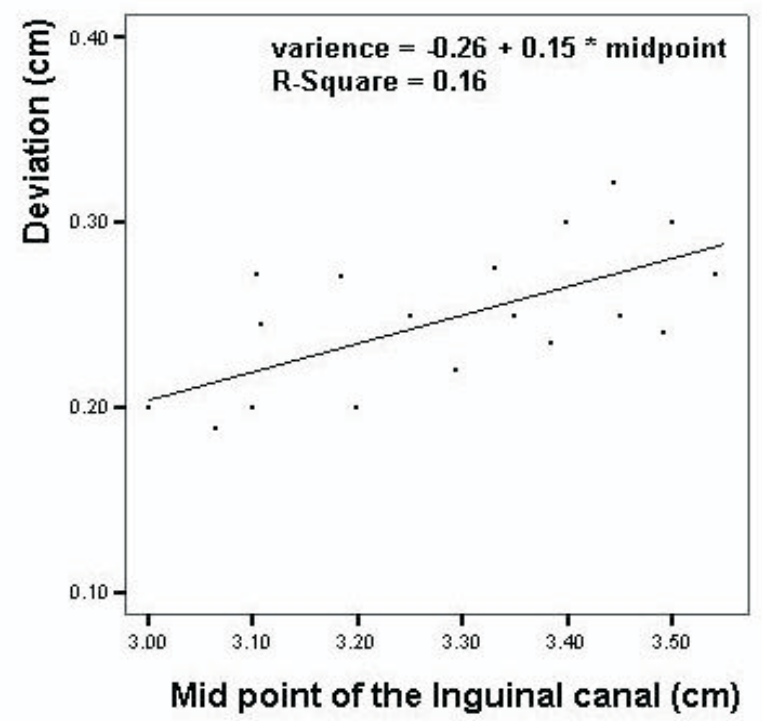

Figure: Linear regression between midpoint of the inguinal canal and the deviation of the deep ring from the mid point.

\section{Discussion}

This paper describes the exact position of the DIR and its relationship to the length of the inguinal canal among Sri Lankan population. We noted that the deep ring is located $2.5 \mathrm{~mm}$ medial to the mid-point. However, Wan and Tan (3) have identified that the true surface marking of the DIR to lie at a mean distance of $9.6 \mathrm{~mm}$ medial to the mid-point of the inguinal ligament landmark and $4.5 \mathrm{~mm}$ lateral to the mid-inguinal point (MIP), approximately one-third of the distance from the MIP. Further, they found no association between the position of the DIR and age, gender, race, body mass index or pelvic habitus. Conaghan et al., (4) found that the DIR was located at a mean of $41 \%$ (95\% confidence interval (CI) $26-56 \%$ ) of the way along the inguinal ligament from the PT towards the ASIS, compared with the $50 \%$ which would be expected from traditional clinical methodology. The DIR was actually found at a mean of $51 \%$ (95\% CI 33-69\%) of the way along a line from the pubic symphysis to the ASIS. Our data are comparable with the above studies.
However, it is worthy to note the following limitations on this study. The study has a limited sample size and a study involving a larger sample is appropriate. It was seen that the actual position of the DIR in those with inguinal hernia, either right or left, was not at the exact midpoint between the PT and ASIS.

\section{References}

1. Sanjay P, Reid TD, Bowrey DJ, Woodward A. Defining the position of deep inguinal ring in patients with indirect inguinal hernias. Surgical and Radiological Anatomy, 2006: 28(2): 121-124.

2. Abeysekara JDA, Shahanavaz H. Body size variability between people in developed and developing countries and its impact on the use of imported goods. International Journal of Industrial Ergonomics, 1989; 4(2): 139-149.

3. Wan WH, Tan ELD. Defining the position of the deep inguinal ring using findings at laparoscopic inguinal hernia repair. Surgical and Radiological Anatomy, 2010; 33(1): 59-63.

4. Conaghan P, Hassanally D, Griffin M, Clark CI. Where exactly is the deep inguinal ring in patients with inguinal hernias? Surgical and Radiological Anatomy, 2004; 26(3): 198-201. 